\title{
GAMIFICATION AS A TOOL OF COMMERCIAL BANK STAFF ECONOMIC ACTIVITY AND STIMULATING
}

\author{
${ }^{1}$ Kazan Federal University, Kazan, Republic of Tatarstan (RUSSIA) \\ ${ }^{2}$ Kazan Federal University, Kazan, Republic of Tatarstan (RUSSIA) \\ Emails: kate.rina_guseva@outlook.com; guzel7011@mail.ru
}

GUSEVA Ekaterina Borisovna1, KHAZIAKHMETOVA Guzel Azatovna²

\begin{abstract}
Human capital is one of the key factors for an organization's success in modern economic conditions. Its formation and implementation depend on the policy of the enterprise in the field of personnel management. Complexity and ambiguity of intra-personal processes - the carrier of human capital determine the necessity of progressive movement in the direction of research of cause-and-effect relations between managerial decisions, corporate environment, and the reaction of personnel to it in the forms of activity on the transformation of the human capital of a personality into the capital of the same name of organization and created value.

Well-known tools of influence on the mentioned processes consist in the realization of mechanisms formal and informal influence and the creation of conditions for successful stimulation of economic activity. Digitization is the modern trend of economic reality in the behavior of the new generation of personalities of staff. This means the devaluation of the tradition of obedience in response to the priorities of freethinking, determination, uncompromising, creativity and independence, the reference point to "easy", "game" attitude to professional and everyday situations. All this determines the daily habits, interests, properties of reaction to the stimuli of human activity determine the necessity to choose alternative methods of influence from the authoritarian ones. In this regard, the development of the theory and methodology of gamification of the employee's activity in the organization is seen as relevant. The presented article is devoted to the research of gamification's methodology of management in a system of non-material stimulation of commercial bank personnel.
\end{abstract}

Keywords: gamification, human capital, personnel management, corporate culture, personnel training, personnel development

JEL:M50

UDC: 005.963.1:004.4

005.42-024.61

005.73:336.71

COBISS.SR-ID 28658441

\section{Introduction}

The modern incentive system is not limited by an application of material motivation.

Managers consider market trends and increasingly turn to instruments of non-material stimulation. This is primarily due to the fact that the young generation evaluates the workplace not only as a source of income, but as a platform for self-realization as well. This means that career growth, professional development and realization of potential are in the 
foreground. Moreover, non-material motivation makes it possible to increase and strengthen employee's loyalty. All these factors make development and research of the system of nonmaterial motivation relevant.

In this regard there are some works which is done in the purposed subject. Vinichenko et $a l$., recognize modern visions about the business gamification by focusing on the gamification application theoretical aspects in various sectors of economy, beside the best practices of gaming techniques application [4]. Also, in other work, gamification success stories identified which have been applied in hospitality and tourism and gamification benefits examined by analyzing the relationship between tourism organizations and three main tourism stakeholders including tourists, tourism employees and local community [5].

\section{Methods}

The methodological basis of the research is systematic, comparative, abstract-logic and economic types of analysis. An algorithm of the presented research is expressed by a chain: system of non-material stimulation of the personnel of PJSC "AK BARS" BANK, problems and prospects - gamification as a way of non-material stimulation of the personnel of PJSC "AK BARS" BANK - an estimation of efficiency of assimilation of methodology of gamification at personnel management of PJSC "AK BARS" BANK.

\section{Results and Discussion}

The system of non-material stimulation of the personnel in PJSC "AK BARS" BANK is based on the following components: corporate culture, personnel training and development, personnel assessment and internal communications development. Employees of various structural subdivisions, departments and other organizational units of the bank are involved in the processes of personnel motivation and stimulation in PJSC "AK BARS" BANK.

According to the data of 2019, the number of employees of PJSC "AK BARS" BANK exceeded 5.5 thousand employees. Most of the personnel of PJSC "AK BARS" BANK are employees younger than 39 years. The total share of this group of personnel in 2019 was $73 \%$ [8]. Based on this information it can be assumed that the implemented stimulation policy should be based on modern trends and the introduction of innovations into the motivation process.

The key trends of globalization are mobility, high speed of decision making, business access to the online platform, flexibility. The coming Digital Revolution requires modernization and improvement of business rules, management models, the introduction of innovations, and increased adaptability. Modern companies must transform from the traditional type of organizations to technological ones in this environment. Thus, PJSC "AK BARS" BANK is now moving to the "banking of the future", completing the transformation of the business model from a classic bank to one of the largest financial technology corporations.

However, for a holistic transformation it is necessary to review the full range of processes of PJSC "AK BARS BANK. One of the most important directions of transformation is the sector of human capital as the basic factor of economic activity itself [12]. The necessary condition for the completion of business transformation is the development of digital strategy [3], integrated not only with the strategic goals of the organization and its business strategy, but with HR-strategy as well. Complex impact allows for achieving desired results.

Nowadays, in research circles, considerable attention is being paid to the theory of generations developed in the 90s by American scientists William Strauss and Neil Howe. This theory, based on the psychology of generations formed by habitats, values and events, 
highlights the value system that governs the behavior of each generation. Thus, Generation $X$ includes people born in 1961-1981, Generation Y - born in 1982-2004, Generation Z - born after 2005. Most of the personnel of PJSC "AK BARS" BANK are people who belong to Generation Y, the share of this group in 2019 was $73 \%$. Therefore, PJSC "AK BARS" BANK needs to form a system of staff motivation based on the features and values of this generation.

Generation Y or Generation Millenials, as it is called, are virtualized for the reason that high technologies are an integral part of their lives. This generation is focused on quick results, values self-development and self-realization, meaningful tasks and flexibility of approach. Career building in the traditional way is of little interest to them, and status and positions are not the end in themselves. Generation Y considers the opportunity to do what they love to do, drive tasks, work in a team, have an experienced mentor, and maintain a balance between work and personal life which are the most important aspects of their professional development [13]. Also, millenniums value flexible working hours, lack of attachment to the workplace, the informality of communication.

The banking sector has its own specifics, so not all trends in the motivation of millenniums will be reflected in the motivation of bank employees. Thus, for example, the main part of activities in the bank is work with documents, which is strictly regulated and formalized as a rule, as well as communication with clients, which also does not allow an informal style of communication. However, many of the existing trends can be adapted to the peculiarities of this business.

Today, a new trend of millennium management is gaining more popularity, which consists of the use of game elements in non-game processes and which is called the term "gamification". The term was used first by American programmer and inventor N. Peling in 2002, and then by Canadian consultant G. Ziechermann in 2003 [10]. Gamification was understood as the process of applying game thinking and using game dynamics to involve the audience in the process of solving problems.

This term has synonyms now, among which are: gambling, gaming and game mechanics in motivating staff. The term "gambling" was used by the Center for Educational Developments of the Moscow School of Management Skolkovo during one of the presentations. The term "playfulness" meant the use of game approaches to actively involve students in the process of mastering new skills and competencies. The term "playfulness" is not new and has been used by Russian teachers in educational methods for a long time. Fatherland representatives of pedagogy L.S. Vygotsky and G.P. Shchedrovitsky paid special attention to the specifics of the game and game activity in education in their works, noting the high efficiency of this method.

The key feature of gamification is the use of specifics and features of computer games and video games, which allows to achieve greater involvement of Generation Y employees in their professional activities [9].

Gamification is an excellent non-financial motivation tool that can increase employees' efficiency, encourage them to achieve better results and bring the team together. Looking at the gamification, it is necessary to highlight the main features of this tool. The main aspects of gamification should include: dynamics, mechanics, aesthetics and social impact. Dynamics manifests itself in the use of game scenarios that involve users in the process and encourage a quick response to events. Gamification mechanics is represented by the use of game elements of encouragement - virtual awards, statuses, virtual goods. The aesthetics are represented by creating a general impression of the gameplay, creating an emotional atmosphere. Social interaction within the framework of gamification should include the use of various game techniques that can provide active interaction of users.

Within the framework of improvement of the system of non-material stimulation of the personnel of PJSC “AK BARS" BANK proposes to apply IT-technology tools that meet the trends of digitization and the needs of Generation Y employees, which make up $73 \%$ of the 
bank's personnel. In order to develop the motivation system, it is proposed to improve the corporate portal AkBars Life available at PJSC "AK BARS" BANK and expand the functionality of this tool in the following areas:

1. Creation of a corporate social net;

2. Introduction of chat bots;

3. Achieving transparency of the employee's performance indicators and the efficiency of their work;

4. Introduction of gamification into HR management processes.

Implementation of these measures will improve internal communication processes; improve the interaction between employees of different departments and subdivisions. In addition, the introduction of chat bots will significantly simplify some of the routine processes and will automate some of them. The openness of performance indicators of the employee will allow to develop healthy competition, which will lead to increased productivity. The introduction of gamification into HR management processes will allow making routine processes more interesting and increasing the level of personnel involvement.

The introduction of gamification into staff incentive processes is caused, in particular, by the acute need of the organization for innovations. It is connected with the fact that innovations are the factor of maintenance and increase of competitiveness today [11].

Gamification allows to make the process of generation and introduction of innovations more exciting by increasing the level of involvement of employees in the processes of the organization. Thus, by means of gamification each employee has an opportunity to contribute to the development of the organization and to see the assessment of this contribution from colleagues and management staff. This satisfies the employee's need for recognition, a sense of importance and value for the work performed. In addition, gamification can create a specific culture of employee involvement, turning routine operations and tasks into interesting opportunities.

Introduction of gamification into the personnel motivation system of PJSC "AK BARS" BANK is connected, first of all, with the necessity to consider the features of the generation of millenials occupying the largest share in the structure of the bank's personnel. According to the research of the British sociologist Richard Bartle, the degree of impact of gamification on different generations differs: on generation $\mathrm{X}-17 \%$, on generation $\mathrm{Y}-51 \%$, on generation $\mathrm{Z}$ $-32 \%$ [1]. Generation Y has the highest susceptibility to gamification methods. Among the personnel of PJSC “AK BARS” BANK there are no employees of Generation Z yet, however, in the coming years the situation will change, as the bank pursues an active policy of attracting young specialists and closely cooperates with universities.

Building an effective policy for managing staff incentives, it is necessary to consider the long-term perspective. This means that the introduction of gamification will be relevant and beneficial for the interaction with staff today and tomorrow.

According to the research conducted by Protection Technologies LLC, which provides business automation services, corporate social nets can improve a number of organizational performances [6]. Simplification of access to information by $77 \%$ occurs through the creation of an internal database of contacts of employees, information tape, which includes information from management and employees, the introduction of chat bots. For example, the use of chat bots makes it possible to simplify routine operations, which include obtaining information about the necessary documents package for applying for the vocation or sick pay, information about career opportunities or upcoming events, training programs and evaluation procedures. This saves HR department staff time and staff time spent on communications to find the information they need.

The availability of knowledge from internal experts is increasing significantly thanks to the introduction of a distance learning system and the publication of useful materials by staff. The 
48 per cent reduction in communication costs is due to the use of effective online communication in dialogue and chat rooms. Employees are becoming more mobile; communications are faster and more accessible. The 68 per cent reduction in travel costs is due to the elimination of the need to move staff because of improved communications between staff members and the ability to monitor certain tasks in real time. Satisfaction of employees increases due to the use of new and better understood technologies, faster feedback, transparency and availability of information.

The use of a corporate social network will also increase employee productivity when performing standard routine tasks. This is the result of uniform release of time when interacting with e-mail - on average $7.5 \%$, when searching for information $-6 \%$, when working together $-4.5 \%$ and the performance of specific tasks $-5 \%$. Thus, productivity at performance of the given operations increases on average on $24,4 \%$. This is a significant growth, which testifies to the proven effectiveness of the introduction of corporate social network in the staff incentive system.

Simplifying access to information reduces the time required to conduct operations. As a result, there is an increase in the speed of implementation of operations and subsequently increases productivity. On the basis of the data given above it is possible to speak about an increase in productivity of employees on 20-25\%. It means that PJSC "AK BARS" BANK will be able to reduce the number of staffs by 1000 staff units, which will lead to savings of the bank's payroll by 65.7 million rubles [7]. The introduction of gamification into the employee incentive system is an important tool of increasing motivation to work by improving a number of indicators.

Above all, gamification helps to increase employee engagement. This is because gaming engagement techniques make the job more engaging, dynamic. Stressful and routine reduce.

Thus, according to LLC "Protection Technologies", after gamification implementation the average increase in the employee engagement rate is $25 \%$. To date, the level of personnel involvement of PJSC "AK BARS" BANK is 61\%, while in PJSC "Sberbank" this indicator in 2018 was $75 \%$. Based on this, we can assume that the increase in this indicator is an urgent task for PJSC "AK BARS" BANK. Thus, as a result of the introduction of gamification, the level of involvement will increase by $25 \%$ and will amount to $76.2 \%$.

Introduction of gamification in the process of adaptation of new employees will improve this process qualitatively. Firstly, employees will have an opportunity to get acquainted with the mission, goals and corporate values of the company rather quickly. Secondly, gamification of this process will simplify the process of adaptation in the corporate social net. A newcomer will have an opportunity to get acquainted with all possibilities of the network, and as a reward for passing the program will receive bonus points.

Introduction of gamification in this process will reduce the number of dismissals due to the difficulties with adaptation by $7 \%$ - this conclusion was reached by experts of McKinsey Global Institute as a part of the study of social technologies in the economy [2]. Today the staff turnover in PJSC "AK BARS" BANK is $25.6 \%$, and dismissals due to difficulties with adaptation make up $12 \%$ of the total number. In 2019 the number of dismissals due to difficulties with adaptation was 167 . This figure is quite high, which means that measures should be taken to reduce its value. As a result of the introduction of gamification, this figure may drop to $11.16 \%$. In numerical terms, the number of dismissals due to adaptation difficulties will decrease by 12 . The economic effect can be estimated by calculation of funds spent on hiring and dismissal of one employee of PJSC "AK BARS" BANK. The data are presented in the Table 1. 
Table 1. Economic effect of introducing gamification in adaptation of new employees

Continuous improvement of staff skills in the implementation of gamification is achieved through the fact that employees receive additional motivation to develop their skills and competencies. Management, in its turn, gets the opportunity to manage this process effectively.

Improvement of intra-corporate interaction and improvement of the psychological climate in the team is achieved as a result of the formation of a comfortable social environment, prompt provision of feedback to employees and rapid integration of employees into the ongoing processes.

The creation of the Bank of Ideas as part of the corporate social net will improve the processes of interaction between managers and employees increase the initiative of staff and will simplify the process of submission and proposals for optimizing existing processes in the organization and ideas for implementing new ones. These practices are used by leading organizations around the world and help increase staff involvement in the organization's activities. According to statistics, the introduction of gamification increases the number of proposals submitted by employees by $68 \%$ and the number of implemented proposals by 25\%. To date, employees of PJSC “AK BARS” BANK submit about 150 proposals per year, of which no more than 10 are implemented. This is an extremely small figure. The introduction of the gamified Ideas Bank will allow to development of initiative and participation in the staff of the organization. Thus, as a result of the introduction of gamification, the number of submitted proposals may increase to 252 , and the number of implemented initiatives to 15 .

Implementation of a corporate social net with gamified tools is quite costly. However, due to the fact that PJSC "AK BARS" BANK already has a corporate portal AkBars Life, the cost of implementation will be lower. It is expedient to apply to outsourcing and purchase a readymade solution, which will be installed on the existing server base. The cost of the ready solution is presented in the Table 2.

Table 2. Program implementation costs, RUR

\begin{tabular}{cc}
\hline Indicator name & Forecast \\
\hline Cost of the decision per 1 employee & 2340 \\
\hline Total solution cost & 12870000 \\
\hline Cost of setting & 150000 \\
\hline Total & 13020000 \\
\hline
\end{tabular}

It is necessary to calculate the economic efficiency to assess the feasibility of implementing the proposed solutions in the processes of stimulating the labor activity of the personnel of PJSC "AK BARS" BANK. For this purpose, the ROI indicator is used. The calculation data are presented in the Table 3. 
Table 3. Cost-effective implementation of corporate social net with gamification tools

\begin{tabular}{cc}
\hline Category & Costs \\
\hline Reduced search costs as a result of resignations due to difficulties in adaptation & $455.028 \mathrm{rub}$ \\
\hline Savings on bank salary fund & $65.700,000 \mathrm{rub}$ \\
\hline Costs of implementing the program & $13.020,000 \mathrm{rub}$ \\
\hline ROI & $408 \%$ \\
\hline Return on investment & 1 year \\
\hline
\end{tabular}

Social and economic efficiency of introduction of corporate social network with gamification tools into the system of labor activity stimulation of PJSC "AK BARS" BANK personnel is presented in the Table 4.

Table 4. Socio-economic efficiency of proposed activities

\begin{tabular}{|c|c|}
\hline Social effectiveness & Economic efficiency \\
\hline 1. Increasing the level of personnel involvement & 1. Increasing labor productivity \\
\hline 2. Increasing the initiative of staff & 2. Decrease in costs related to staff turnover \\
\hline 3.Increased level of staff satisfaction & 3. Reducing communication and travel costs \\
\hline
\end{tabular}

\section{Summary}

Based on the assessment of efficiency of the proposals on development of the system of personnel stimulation of PJSC "AK BARS" BANK, the following conclusions can be made:

1. Introduction of corporate social net will allow to ensure the effectiveness of information flows of the organization, which in its turn, will simplify access to the necessary information by $77 \%$, reduce communication costs by $48 \%$, reduce travel expenses by $68 \%$ and increase staff satisfaction level by $56 \%$. It will also promote a sense of ownership of the organization and commitment to corporate values.

2. As a result of implementation of the corporate social net will be increase in productivity by $20-25 \%$. As a result, PJSC "AK BARS" BANK will be able to reduce the number of staffs by 1,000 staff units, which will result in savings of the payroll of 65.7 million rubles.

3. Application of gamification tools in the corporate social net has a long-term perspective, as this toolkit is highly effective in interaction with both Generation Y and Generation Z, which in the near future will constitute a high proportion of the PJSC "AK BARS" BANK personnel.

4. The introduction of gamification will significantly increase the level of involvement of employees of PJSC "AK BARS" BANK. According to statistics, the average growth of this indicator as a result of gaming methods is $25 \%$. Thus, the level of staff involvement will be $76.2 \%$.

5. Application of gamification in adaptation of new employees allows to achieve high efficiency of this process, as well as to reduce the number of dismissals due to difficulties in adaptation of new employees by $7 \%$, which will reduce the cost of personnel search by 455,028 rubles per year.

6. Application of ideas by the Bank within the framework of the gamification of corporate social net will allow to increase initiative and involvement of employees of PJSC "AK BARS" BANK. The number of proposals submitted by employees may increase by $68 \%$, while the number of implemented initiatives may increase by $25 \%$.

7. The total economic effect from the development of proposals may reach about 53 million rubles per year. 


\section{Conclusions}

The presented article is devoted to the research of gamification's methodology of management in a system of non-material stimulation of commercial bank personnel.

On the basis of the conducted research, it is possible to make a conclusion that the introduction of the corporate social net into the system of non-material stimulation of the personnel of PJSC "AK BARS" BANK with application of gamification methods can become the catalyst of the organization development and qualitatively improve a number of indicators characterizing the level of motivation and involvement of the personnel.

\section{Acknowledgements}

The work is performed according to the Russian Government Program of Competitive Growth of Kazan Federal University.

\section{REFERENCES}

[1] Bartle, R. Gamification impact study on employee engagement. Sibirix URL: https://blog.sibirix.ru/2014/07/10/ bartle/

[2] Guseva, E., \& Haziahmetova, G. (2020). Analysis of the system of non-material stimulation of Ak Bars bank personnel. IV All-Russian Forum "Economy in a Changing World".

[3] Nagibina, N.I., \& Shchukin, A.A. (2017). HR-Digital: Digital technologies in human resource management. Internet journal "SCIENCE 9-1", pp. 24-25.

[4] Vinichenko, M. V., Melnichuk, A. V., Kirillov, A. V., Makushkin, S. A., \& Melnichuk, Y. A. (1970). Modern views on the gamification of business. The Journal of Internet Banking and Commerce.

[5] Negruşa, A. L., Toader, V., Sofică, A., Tutunea, M. F., \& Rus, R. V. (2015). Exploring gamification techniques and applications for sustainable tourism. Sustainability 7(8), pp. 11160-11189.

[6] Official page of "Protection Technologies" URL: https://pryaniky.com/ru-pryaniky-as-service/rucorporate-network/

[7] Research by McKinsey Global Institute "Social economy: revealing value and productivity through social technology" URL: https://www.mckinsey.com/industries/technology-media-andtelecommunications/our-insights/the-social-economy

[8] The official website of OJSC Ak Bars Bank URL: https://www.akbars.ru/

[9] Velikikh, P., \& Zaryanova, Y. (2015). HR-gamification: how to use gaming technology in personnel management. Innovation Science 10, pp. 53-54.

[10] Vernah, K., \& Hunter, D. (2015). Involve and conquer. Playful thinking in the service of business. Publishing house Mann, Ivanov, Ferber, pp. 251-253.

[11] Vertinova, A., \& Borodina, D. (2019). Digitalization of personnel management. Business and economics 3, pp. 13-16.

[12] Zainutdinova, E., \& Khaziakhmetova, G. (2019). Retrospective study of approaches to the definition of «human capital». SCOPUS-2019-11-8-SID85073441939.

[13] Zemskova, M., \& Krasnova, M. (2016). Introduction of gamification into the process of motivation of Y generation personnel. Economic sciences 8(26), pp. 46-49.

\section{Article history:}

Received 25 May 2020

Accepted 30 Juny 2020 